\title{
Enseñanza de la Botánica en un contexto local: una propuesta didáctica basada en el naranjo morisco de Hornachos Teaching Botany from a local context: a didcatic proposal based on Hornacho's Moorish orange tree
}

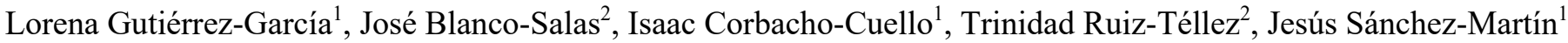 \\ lorenagg@unex.es, blanco_salas@unex.es, icorbacho@unex.es, truiz@unex.es, jsanmar@unex.es \\ ${ }^{1}$ Didáctica de las Ciencias Experimentales y \\ Matemáticas \\ Universidad de Extremadura \\ Badajoz, España \\ ${ }^{2}$ Biología Vegetal, Ecología y Ciencias de la Tierra \\ Universidad de Extremadura \\ Badajoz, España
}

\begin{abstract}
Resumen- Se propone una unidad didáctica en la que se utiliza una variedad de naranjo propio de la localidad donde radica el estudio, el naranjo morisco de Hornachos (Hornachos, España), con el fin de aumentar el interés por el aprendizaje de la Botánica y del valor y riqueza de su propio entorno. Se pretende evaluar la importancia del empleo de contenidos originarios de "lo local", del patrimonio etnográfico propio de cada lugar. Para ello se lleva a cabo una unidad didáctica basada en los contenidos oficiales pero en la que se introducen actividades y ejemplos centrados en el naranjo morisco de Hornachos. Para evaluar las posibles ventajas, se tomará un grupo control en el que se impartirá una unidad de diseño similar pero tomando como referencia otras especies más genéricas. Previo al desarrollo de dichas unidades se realizará un test para evaluar los conocimientos y motivación previos en ambos grupos. Posteriormente a la experiencia didáctica, se realizará un nuevo test que permitirá comparar la evolución de cada grupo y entre ellos. Se esperan obtener resultados positivos que relacionen la propuesta didáctica con el aumento del aprendizaje significativo y la puesta en valor de la riqueza de su propio medio.
\end{abstract}

\section{Palabras clave: Educación y Cultura, Etnobotánica; Educación Secundaria.}

\begin{abstract}
A didactic unit is proposed in which Moorish orange tree, a local variety of orange tree characteristic of the town where the study is carried out (Hornachos, Spain), is used as a tool to increase interest in learning botany and the value and natural wealth of the local environment. It is intended to evaluate the importance of using "local contents", taking advantage of the ethnographic heritage of each place. To this end, a didactic unit is implemented based on the official contents but in which activities and examples focus on the Moorish orange tree of Hornachos. To assess the possible advantages, a control group is taken, in which a similar design unit is delivered, but using as reference other more generic species. Prior to the development of these units, a test is carried out to evaluate previous knowledge and motivation in both groups. After the didactic experience, a new test is delivered to allow to compare the evolution of each group and between them. Positive results are expected to relate the didactic proposal to the increase in meaningful learning and the value of the wealth of its own environment.
\end{abstract}

Keywords: Culture and Education; Ethnobotany; Secondary School.

\section{INTRODUCCIÓN}

La importancia de la Ciencia en la sociedad actual conlleva la necesidad de formar científicamente a los ciudadanos (Solbes, Montserrat, \& Furió, 2007), no solo para garantizar la formación de futuros científicos sino para que cualquier persona tenga la capacidad de construir una opinión crítica y fundamentada de los avances que se producen, sus limitaciones y aplicaciones (Comisión de las Comunidades Europeas, 2006). Sin embargo, si nos centramos en España, aunque la inquietud por la Ciencia ha aumentado en los últimos años (Esteve \& Solbes, 2017), sigue generando desinterés en gran parte de la sociedad y esto unido a los problemas de género y la metodología de enseñanza de las mismas, se refleja en el alumnado (Esteve \& Solbes, 2017; Solbes et al., 2007).

Existen abundantes datos que revelan que los alumnos no aprenden muchos de los conceptos científicos debido a que se alejan de su conocimiento cotidiano (Pozo, 2000). Es necesaria una reestructuración en la metodología docente (Delgado et al., 2016) que logre una mayor cercanía e implicación del alumnado en la Ciencia (Vilches, A.; Gil, 2008).

Si nos centramos en los niveles educativos preuniversitarios, una de las Ciencias en la que los alumnos muestran dificultades para su aprendizaje es la Biología (Wai, H. O.; Khine, 2020), y dentro de ella la Botánica constituye una de las áreas en las que demuestran mayor desconocimiento (Rivero-Guerra, 2019) .

Ante esta situación, se propone la puesta en práctica de una unidad didáctica, dentro del programa de la asignatura Biología y Geología en el nivel $1^{\circ}$ de la Enseñanza Secundaria Obligatoria (E.S.O.), en el Instituto de Enseñanza Secundaria (I.E.S.) "Los Moriscos" de Hornachos (Badajoz, España), que incluye en sus contenidos materiales cercanos, en este caso concreto, una variedad de naranjo, el naranjo morisco propio de la localidad de Hornachos (Badajoz, España). Está diseñada para trabajar los contenidos básicos establecidos (Junta de Extremadura. Consejería de Educación y Empleo, 2016) pero tomando como referencia lo local, haciendo más identificables 
los conceptos por parte del alumnado y dotando de mayor importancia a la agrobiodiversidad autóctona. Se trata de una experiencia docente evaluable con estándares internacionales, que pretende la mejora del aprendizaje de la Botánica a la vez que pone en valor el patrimonio propio.

El objetivo de este trabajo es dar a conocer el diseño de una metodología que implementa el uso de materiales cercanos en la enseñanza, con el fin de contribuir a la mejora de la enseñanza-aprendizaje de la Ciencia, promoviendo a su vez el empoderamiento del medio rural y la defensa de su riqueza cultural y natural. Para ello, se analizan las diferentes etapas y se exponen las ventajas que supondrían su puesta en práctica.

\section{CONTEXTO}

\subsection{El entorno.}

En primer lugar se realizó una selección del lugar donde llevar a cabo la investigación. Era necesario un Centro perteneciente a un entorno rural rico en conocimientos ligados al entorno natural.

Hornachos es una localidad de la provincia de Badajoz con varias particularidades. Esta población cuenta con un pasado histórico-cultural muy rico (Gutiérrez-García, LabradorMoreno, Blanco-Salas, Monago-Lozano, \& Ruiz-Téllez, 2020) y se asienta en los límites del área protegida "Sierra Grande de Hornachos", con varias catalogaciones de protección tanto regionales -Zona de Interés Regional (ZIR)- como internacionales -Zona de Especial Protección para las Aves (ZEPA), Lugar de Importancia Comunitaria (LIC) y Zona de Especial Conservación (ZEC)- incluyéndose en la Red Ecológica Europea Natura 2000 (Junta de Extremadura, n.d.). Estudios previos avalan gran riqueza de plantas silvestres y cultivadas, a las que se asocian numerosos usos y saberes tradicionales (Blanco-Salas, Gutiérrez-García, LabradorMoreno, \& Ruiz-Téllez, 2019).

El I.E.S. "Los Moriscos" es el único Centro de Enseñanza Secundaria presente en Hornachos y con el que ya se mantenía una relación por estudios previos llevados a cabo en él. Por tanto, se consideró que era idóneo para esta nueva propuesta de intervención.

\subsection{El naranjo morisco.}

El material sobre el que se centra esta propuesta es una variedad local de naranjo, propio del pueblo de Hornachos, que es conocido por los locales como "naranjo de la tierra" y al que hemos determinado denominar "naranjo morisco de Hornachos" con el fin de concretar más en su definición (Figura 1).

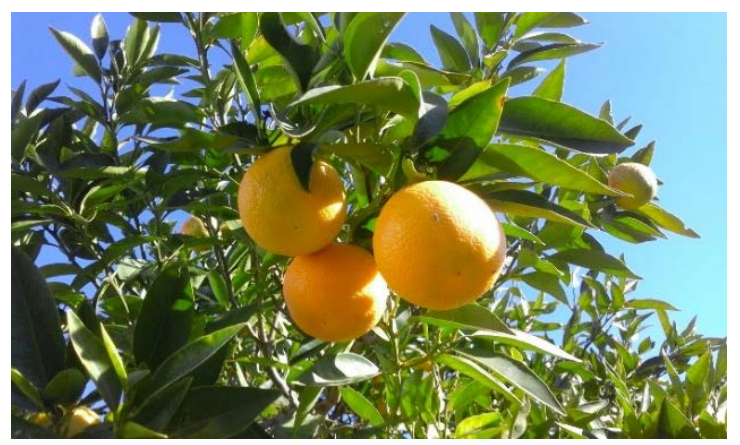

Figura 1.
Previo al diseño de la unidad didáctica que nos ocupa, fue necesario realizar un análisis del contexto en lo referente a la presencia y distribución de ejemplares de este naranjo en la localidad, con el objetivo de determinar el estado de conservación de dicha variedad en la localidad y con los datos que se obtuvieran, sentar las bases del estudio que nos ocupa. Para ello se partió de una lista de huertas moriscas realizada para el Proyecto "Huertas moriscas, agricultura histórica" que se desarrolló durante varios años en la primera década del siglo XXI (ASIRIOMA, n.d.) en la que se recogían los datos relativos a un total de 32 huertas presentes en la localidad de Hornachos.

A partir de este listado base, se elaboró una nueva tabla Excel en la que se tuvo en consideración los siguientes parámetros: Nombre de la huerta, Polígono, Parcela 1, Parcela 2, Longitud, Latitud, Superficie Total (ha), Propietario/a, Agricultor/a, Teléfono Y Dirección de la huerta. Además, se añadieron: $\mathrm{N}^{\mathrm{o}}$ huerta, Zona, $\mathrm{N}^{\mathrm{o}}$ naranjos moriscos presentes.

Con ayuda de Sistemas de Información Geográfica, se localizaron cada una de las huertas y se hicieron visitas directas a lo largo de dos semanas, con el fin de contabilizar los ejemplares de naranjo morisco presentes en cada terreno, mediante la conversación con el dueño/agricultor "in situ", pudiendo verificar que la información era veraz. En algunas ocasiones fueron los presentes en huertas vecinas los que daban la información que se buscaba. También se realizaron llamadas telefónicas y visitas a la dirección del propietario/a.

De este modo se obtuvo un mapeo general bastante significativo de la prevalencia de esta especie con un total de 17 huertas y unos 120 ejemplares de naranjo morisco en la localidad de Hornachos y que se recoge en la Tabla 1.

Tabla 1.

\begin{tabular}{llc}
\hline Zona & \multicolumn{1}{c}{ Nombre de la huerta } & $\begin{array}{c}\mathrm{N}^{\circ} \text { naranjos } \\
\text { moriscos }\end{array}$ \\
\hline 1 & La Virgen (Cerro Las Cruces) & 1 \\
1 & Los Remedios & $>1$ \\
1 & Cañada de las Cruces & 10 \\
2 & Huerta del Negro & $30-40$ \\
3 & La Irine & 4 \\
4 & Los Cristianos & $>1$ \\
4 & San Francisco (Los Cristianos) & 11 \\
4 & San Francisco (Los Cristianos) & 0 \\
5 & Los Cristianos & 4 \\
6 & La Fuente Los Moros (Molla y Palomares) & 2 \\
6 & San Francisco (Los Cristianos) & 3 \\
6 & Desconocido & 10 \\
6 & Desconocido & 10 \\
7 & Los Huertos & $>3$ \\
8 & Pilar Palomas & $>4$ \\
8 & Huerta del Lute & 3 \\
8 & Desconocido & 10 \\
\hline & &
\end{tabular}




\subsection{El Centro.}

El I.E.S. "Los Moriscos" fue creado en 1997. Se trata de un Centro de titularidad pública al que acuden alumnos de Hornachos y de localidades cercanas.

El alumnado no supera los 400 individuos y procede de familias de nivel socioeconómico bajo, donde las principales fuentes de ingresos proceden de la ganadería, agricultura o la construcción. La inmigración es mínima y no hay grandes diferencias socioculturales. Además, existen pocos casos de Alumnado Con Necesidad Específica de Apoyo Educativo (ACNEAE) y aún menos con Adaptaciones Curriculares Significativas.

La oferta formativa abarca desde los niveles de E.S.O., Bachillerato en las modalidades de Ciencias, Humanidades y Ciencias Sociales, a la Formación Profesional con el Ciclo Formativo de Grado Medio "Atención a personas en situaciones de dependencia".

\subsection{Destinatarios.}

Tras un acuerdo previo con el equipo docente y directivo del Centro, se seleccionó como futuros destinatarios para el desarrollo de la unidad, alumnos de $1^{\circ}$ E.S.O. En concreto dos grupos, de los cuatro existente. Se trata de un total de 36 jóvenes con una media de edad de 13 años.

Se escogió en base al currículo básico establecido (Junta de Extremadura. Consejería de Educación y Empleo, 2016; Ministerio de Educación, 2015) para la materia de Biología y Geología en E.S.O. Los contenidos relativos a Botánica, donde poder encuadrar el naranjo morisco como herramienta de apoyo, se incluyen en el Bloque 3. "La Biodiversidad en el Planeta Tierra" y son abordados en el primer curso del primer ciclo.

\section{DESCRIPCIÓN}

Para el diseño de la unidad y la planificación de su puesta en marcha y evaluación, se definen los objetivos y criterios a seguir, el orden y temporalización de cada una de las fases y el diseño de cada uno de los elementos, configurando una propuesta para el análisis del conocimiento e inquietudes adquiridas por los alumnos relativos a la Botánica, desde una perspectiva etnográfica, cuestionando su interés en relación a otros enfoques más globales. El siguiente paso que se pretende es llevar a cabo su aplicación real, obteniendo resultados que permitan reflexionar sobre las ventajas y desventajas que este diseño puede conllevar.

\section{Resultados}

Tras un primer análisis del contexto y de la realidad del Centro I.E.S. Los Moriscos, se seleccionan 2 grupos de $1^{\circ}$ de la E.S.O., uno Control (Grupo A) y otro Experimental (Grupo B). Se utiliza como criterio de selección el número similar de alumnos y el hecho de que comparten docente para la asignatura Biología y Geología. Esto permite que los resultados que se obtengan tras la puesta en práctica del procedimiento sean comprables. El trabajo con los alumnos se desarrollará en tres fases (Figura 2):

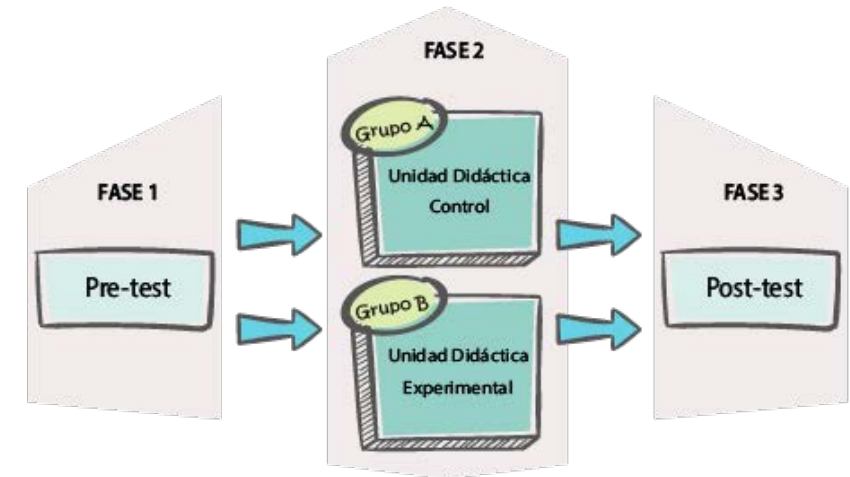

Figura 2.

- En la primera se examinan los conocimientos previos sobre Botánica y Etnobotánica y la motivación hacia el aprendizaje de esta ciencia mediante la aplicación de un Pre-test similar para ambos grupos. Se diseña a modo de cuestionario cerrado donde las preguntas van enfocadas al obtener datos en relación a: aspectos cognitivos, emocionales (Figura 3) y a la visión sobre sostenibilidad.

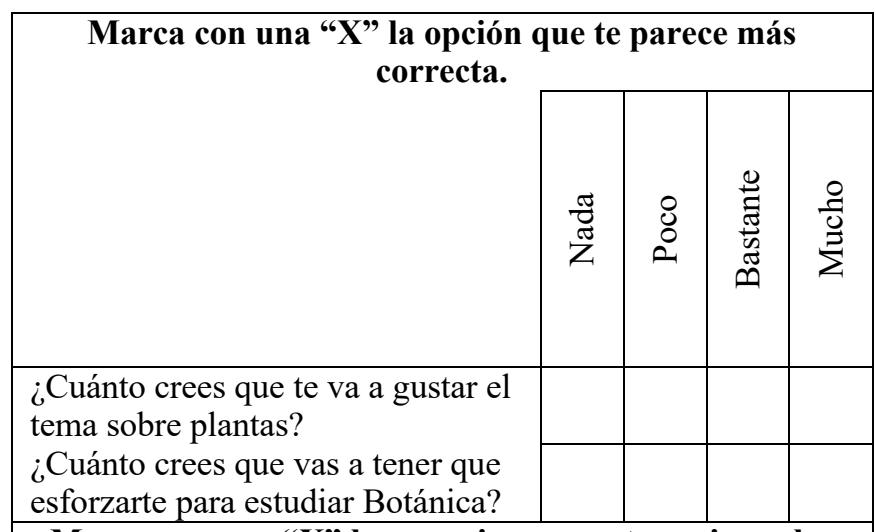

Marca con una " $X$ " las emociones que te sugieren los siguientes contenidos.

El origen y

características de las

plantas

Las plantas con vasos

conductores

Las plantas con

semillas

Las plantas se adaptan

al medio

Las plantas autóctonas

y las no autóctonas

Figura 3. 
- La segunda es la implementación de una unidad didáctica en cada grupo-clase. Se diseñan dos modelos de unidad basados en el libro de texto que se emplea habitualmente: una "Tradicional" y otra "Nueva" donde se incluyen actividades y ejemplos basados en el naranjo morisco, en sustitución de los tradicionales incluidos en el libro mediante el empleo de vídeos - entrevistas con personas mayores de la localidad, explicando los usos e importancia de estos vegetales en la zona. En ambos casos se combinará la enseñanza tradicional con metodologías activas.

- Finalmente se recoge mediante un test final o Posttest, información sobre los conocimientos adquiridos, la motivación y la visión sobre sostenibilidad, con el fin de detectar posibles cambios y evaluar la efectividad de la propuesta. Este cuestionario será el mismo para ambos grupos y a su vez con el Pre-test, pudiendo ser interesante incluir algunas cuestiones más, relativas a los contenidos teóricos de la Unidad, con un carácter más específico a lo visto en el aula.

\section{CONCLUSIONES}

Se prevé que el método presentado genere en los alumnos el aumento de la motivación hacia el conocimiento de la Botánica, que se traduciría, a su vez, en un aprendizaje significativo y en una puesta en valor de la riqueza del entorno cercano, focalizado en este caso en el naranjo morisco.

Creemos que esta metodología es aplicable a muchos otros ámbitos, no sólo de la Biología sino de otras ciencias y que puede permitir dar visibilidad al potencial del conocimiento tradicional y la riqueza natural para mejorar la enseñanza a la vez que se potencia el empoderamiento de la sociedad rural.

\section{AgRADECIMIENTOS}

Este trabajo fue parcialmente financiado por los proyectos de investigación EDU2016-77007-R (AEI/ERDF, EU), GR18004 e IB18004 (Junta de Extremadura y Fondo Europeo de Desarrollo Regional) y las Becas Oportunidad al Talento de la Fundación ONCE.

También hacer constar nuestro agradecimiento al pueblo de Hornachos, a ASIRIOMA y a los alumnos y equipo docente del I.E.S. "Los Moriscos" por su cooperación durante el diseño de este trabajo y la planificación de su futura puesta en práctica.

\section{REFERENCIAS}

ASIRIOMA. (n.d.). Huertas moriscas, agricultura histórica.pdf (p. 92). p. 92

Blanco-Salas, J., Gutiérrez-García, L., Labrador-Moreno, J., \& Ruiz-Téllez, T. (2019). Wild plants potentially used in human food in the protected area "Sierra Grande de Hornachos" of extremadura (Spain). Sustainability (Switzerland), 11(2). https://doi.org/10.3390/su11020456

Comisión de las Comunidades Europeas. Recomendaciones del Parlamento Europeo y del Consejo de Europa sobre las competencias clave para el aprendizaje permanente. , 30.12.2006 Diario Oficial de la Unión Europea § (2006).

Delgado, J., Vallés, C., Gil, C., López, M. A., Verde, A., Allué, J. R., ... Gago, A. (2016). Opiniones sobre la enseñanza científica y dificultades de aprendizaje de las ciencias en maestros en formación. 27 Encuentros de Didáctica de Las Ciencias Experimentales, (October), 713-719.

Esteve, A. R., \& Solbes, J. (2017). El desinterés de los estudiantes por las Ciencias y la Tecnología en el Bachillerato y los estudios universitarios. X Congreso Internacional Sobre Investigación En Didáctica de Las Ciencias, 573-578.

Gutiérrez-García, L., Labrador-Moreno, J., Blanco-Salas, J., Monago-Lozano, F. J., \& Ruiz-Téllez, T. (2020). Food Identities, Biocultural Knowledge and Gender Differences in the Protected Area "Sierra Grande de Hornachos" (Extremadura, Spain). International Journal of Environmental Research and Public Health, 17(7), 2283. https://doi.org/10.3390/ijerph17072283

Junta de Extremadura. Consejería de Educación y Empleo. DECRETO 98/2016, de 5 de julio, por el que se establecen la ordenación y el currículo de la Educación Secundaria Obligatoria y del Bachillerato para la Comunidad Autónoma de Extremadura. , Diario Oficial de Extremadura $\S(2016)$.

Junta de Extremadura. (n.d.). Áreas portegidas de Extremadura. Retrieved June 20, 2021, from http://extremambiente.juntaex.es/index.php?option=com _content\&view $=$ article\&id $=1285 \&$ Itemid $=459$

Ministerio de Educación, C. y D. (2015). Real Decreto 1105/2014. Boletín Oficial Del Estado, 35.

Pozo, J. I. (2000). ¿Por qué los alumnos no aprenden la ciencia que les enseñamos?: el caso de las Ciencias de la Tierra. Enseñanza de Las Ciencias de La Tierra, 8(1), 13-19. https://doi.org/10.26620/uniminuto.praxis.5.6.2005.4849

Rivero-Guerra, A. O. (2019). Impacto de Tres Modelos de Enseñanza de la Asignatura Botánica General sobre el Rendimiento Académico de los Estudiantes. Formación Universitaria, 12(3), 67-80. https://doi.org/10.4067/s0718-50062019000300067

Solbes, J., Montserrat, R., \& Furió, C. (2007). El desinterés del alumnado hacia el aprendizaje de la ciencia: implicaciones en su enseñanza. Didáctica de Las Ciencias Experimentales y Sociales, 21, 91-117. https://doi.org/10.7203/dces..2428

Vilches, A.; Gil, D. (2008). ¿ Qué puede estar contribuyendo al desinterés del alumnado hacia los estudios científicos? Algunas propuestas de solución fundamentadas en la investigación https://doi.org/10.13140/RG.2.1.1305.3681

Wai, H. O.; Khine, S. S. (2020). An investigation into the difficulties of students in learning Biology. J. Myanmar Acad. Arts Sci., XVIII(9). 